\title{
Information security for optoelectronic ergatic system
}

\author{
A.V. Bushma \\ O.S. Popov Odessa National Academy of Telecommunications \\ 1, Kuznechnaya str., 65029, Odessa, Ukraine
}

\begin{abstract}
System representation in view of information security for otoelectronic ergatic means is formed. Analytical description in the matrix form for corresponding systems is received. Properties of the received bidimensional matrix are investigated.
\end{abstract}

Keywords: ergatic system, information defense, digital data, electro-optical converter, matrix description.

Manuscript received 02.02.10; accepted for publication 25.03.10; published online 30.04.10.

\section{Introduction}

Automation of controlling systems, increasing the requirements to their precision, operation speed and information capability in combination with continuous modernization of data reception and transfer technologies are tightly bound with constant and essential increase in information volumes that are processed in controlling and information-communication complexes. At the same time, multi-factorial character of external actions on a technical system and diversity of available ways to obtain an optimal final result implies, in most of cases, presence of an operator when controlling complex objects and technological processes.

As a result, sharply increased are the requirements to quality of information resources and directly to systems for reception, transmission, procession and displaying the data. Disturbance of the main information features - confidentiality, integrity and accessibility results in adoption of mistaken decisions and, as a consequence, is related with casualty-producing capacities and other undesired situations. Therefore, protection of information in an ergatic system is extremely important both at the stage of its creation and in the operational process. Any used methods and means should prevent the leak of protected information as well as unauthorized and unintended influence on it $[1,2]$.

Essential progress in microelectronics provides creation of new and rather accessible means and methods to extract and attack information as well as results in saturation of surrounding medium with diverse electronics. As a result, software-hardware and engineering support in information protection becomes more and more topical. At the same time, organizationallegal methods become less essential. In this situation, the very task of information protection is rather difficult and has no formal methods to solve it. The methodological basis to solve tasks of this class can be provided by the system approach that is based on investigation of an object by using a respective model [2,3]. But models of ergatic optoelectronic systems aimed at investigation of protectability of information circulating in them are absent up to date.

This work is devoted to modeling and analytical description of ergatic optoelectronic digital informationcontrolling systems from the viewpoint how to protect the processed data.

\section{Conception}

In a general case, modeling this system consists of creation of some its image corresponding (with an accuracy up to the purposes of modeling) to the investigated object in order to obtain the necessary characteristics of a real system by using this model. The purpose determines principles for creation of this model, which are realized as being based on the choice of a respective set of criteria for system classification. In our case, the task is to create a generalized model for a human-machine optoelectronic system from the viewpoint how to protect information circulated in it.

Here, to simplify the task we shall consider the operator as a determinate element, assuming that his parameters for processing the coming communications and controlling actions on other system elements are rigorously determined [4-7]. Our analysis of the used principles for realization of mathematical models enabled us to formulate the required set of main criteria for description of the investigated optoelectronic ergatic system [8-11].

1. A model-prototype relationship related with a level of characteristics that are determined using this model. Here, one should separate general and partial models that are created to determine generalized and 
local system parameters, respectively. It is obvious that in our case we shall construct some general model that will enable to study security characteristics for the system in the whole.

2. A way for modeling that is determined by the approach put into the basis of the built model. With account of this sign, all the models can be separated by two groups: analytical and statistical. The first group is realized in the form of mathematical dependences that allow analytical determining the necessary system characteristics. While statistical modeling is based on processing the results of multiple imitation of the necessary dependences for characteristics on external and internal system parameters. For the level of a generalized model representing the ergatic system, we shall use the analytical way for modeling as the most efficient in estimation of potential threats to this system as well as in development of methods to overcome them.

3. The system type mainly dependent on the level of certainty of interrelations between system parameters both analyzed and influencing on them, The same concerns parameters of system elements and external medium. When modeling in accord with this criterion, it is important to ascertain whether this system is determinate or stochastic. As to the first type, all the dependences are rigorously and uniquely determined, while to the second type there is an essential influence of random factors on them. In our case, the certainty in element parameters used to reveal the most essential threats to the optoelectronic system assumes application of a determinate model.

4. A way of information representation, which depends on values of variables and character of time running in the system. Here, continuous, discrete and combined models can be realized. Since the constructed system representation uses digital structures, the object parameters and time in the model will possess a discrete character.
5. Linearity of the system related with validity or invalidity of the superposition principle. In the former case, the linear model is used, but in the latter one - non-linear. At the used level of generalization, we shall consider the ergatic optoelectronic system as the linear one.

\section{Model}

Let us form some general analytic model for linear determinate discrete optoelectronic system. As a principle for separation of system objects, we shall use the criterion of unchangeability in the information representation form or functions for data conversion. In this approach, our study should comprise all the information structure from the communication source up to the operator who is an ultimate data receiver $[12,13]$.

It is a most essential to analyze influence of channels for information transfer between system elements on processing the signals and their transformation, as well as interaction of its elements with surrounding medium. This representation of the ergatic system is depicted in Fig. 1, where: 1 is the initial information converter, 2 - channel for data transfer, 3 system for information processing, 4 - electro-optical converter, 5 - channel for communication with the operator, 6 - system operator, 7 - surrounding medium.

The element 1 of the system is a form-maker for the information signal that is displayed after respective processing and aimed at further reception by the operator in the form of a message $S$. In a general case, its function is to receive the necessary data $I_{X}$ from surrounding medium 7 and transform them into the signal form $D_{X 0}$ convenient for transmission through the connection channel 2. This channel is duplex and provides two-way exchange of messages between the converter 1 and system 3 for processing information. The element 3 processes information $D_{X}$ received via the connection channel 2 and forms the signals $E_{S}$ to control the electro-optic converter 4.

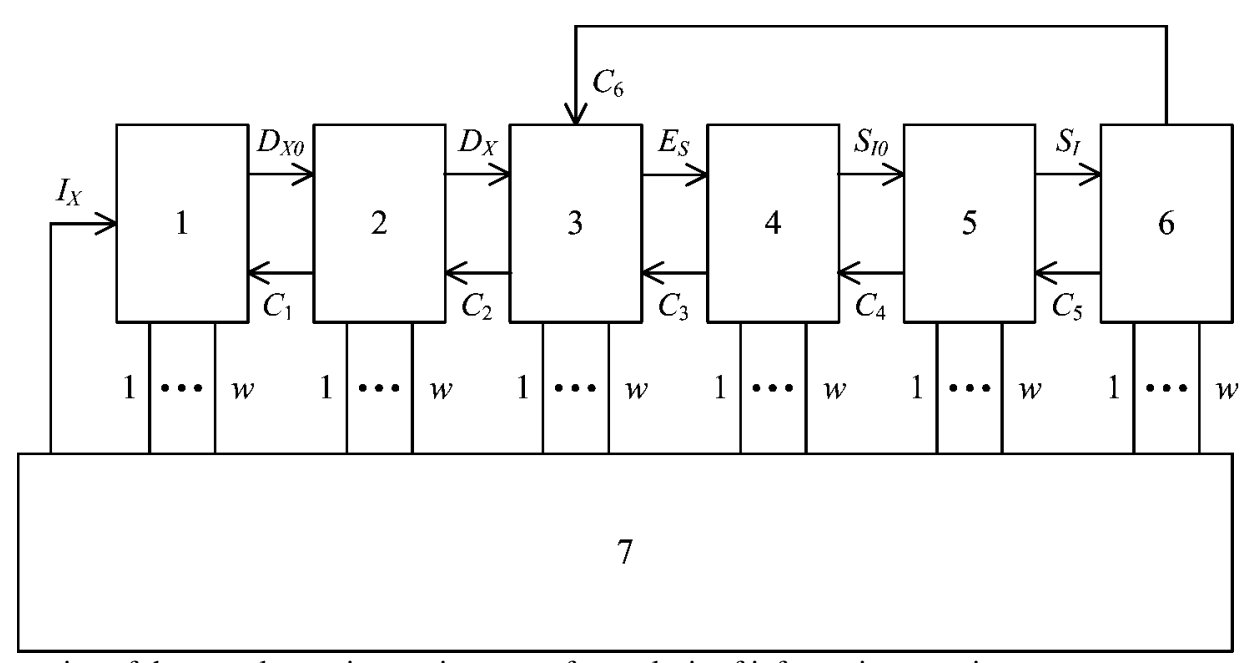

Fig. 1. Representation of the optoelectronic ergatic system for analysis of information security. 
The latter converts the electrical signals $E_{S}$ into the visual message $S_{I 0}$, intended for man reception. The converter 4 via the channel 5 is connected with the operator 6. Using this channel, the optical information signal is received by the operator in the form $S_{I}$. In his turn, the operator 6 has the possibility to act on apparatus means in the system. The corresponding interunit relations $C_{i}$ (where $i=1,6$ ) describe control over the elements 1 to 5 in this system by the operator 6 . Surrounding medium 7 provides multi-channel ( $w$ channels) complex action on all the elements of the ergatic system. Present here are both non-information actions and those carrying a threat.

The structural matrix $\mathbf{M}^{\mathrm{SES}}$ of the considered representation for the ergatic system depicted in Fig. 1, from the viewpoint of information protection, is quadratic and may be written in the following form

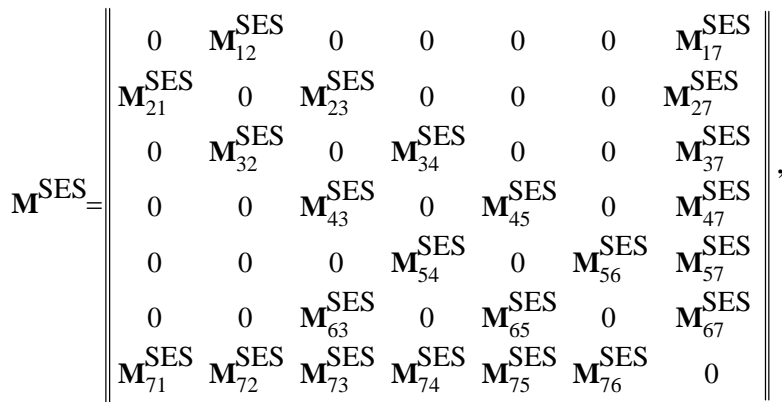

$n_{i} \times m_{j}$ that describe relations between $i$-th and $j$-th system elements and take the following look

$\mathbf{M}_{i j}^{\mathrm{SES}}=\left\|\begin{array}{cccc}M_{11}^{\mathrm{SE}} & M_{12}^{\mathrm{SE}} & \ldots & M_{1 m_{j}}^{\mathrm{SE}} \\ M_{21}^{\mathrm{SE}} & M_{22}^{\mathrm{SE}} & \ldots & M_{2 m_{j}}^{\mathrm{SE}} \\ \vdots & \vdots & \vdots & \vdots \\ M_{n_{i} 1}^{\mathrm{SE}} & M_{n_{i} 2}^{\mathrm{SE}} & \ldots & M_{n_{i} m_{j}}^{\mathrm{SE}}\end{array}\right\|$,

where $\mathbf{M}_{k l}^{\mathrm{SES}}=0 \vee 1$ is the binary value that corresponds to the relation between the $k$-th output of the $i$-th system element and the $l$-th input of its $j$-th element and is equal to unity when this relation is present or zero when this relation is absent for $k=\overline{1, n_{i}}$, $l=\overline{1, m_{j}}$.

The ergatic system shown in Fig. 1 and described with the matrixes (1) and (2) is closed. It determines the absence of a further (higher) level of representation that could include the investigated system as an element of a lower level. It is obvious that in this approach all the objects influencing information security are really represented in this analysis. That allows limiting this consideration and modeling possible threats to information with the determined level for this system.

\section{Conclusion}

The obtained expressions (1) and (2) reflect operation of technical means aimed at visual transfer of data to the operator, with account of essential (from the viewpoint of information security) elements and relations, and allow determining the most probable sources of threats in the system as well. The offered mathematical apparatus is a convenient analytical tool to investigate information security in ergatic systems as well as to choose the most efficient methods for information protection, which is extremely important when creating controlling and information-communication complexes of both general and special purposes.

\section{References}

1. N.V. Grishina, Organization of a Complex System for Information Protection. Helios ARV, Moscow, 2007 (in Russian).

2. A.A. Malyuk, Information Security: Conceptual and Methodological Basics for Information Protection. Goryachaya liniya-Telekom, Moscow, 2004 (in Russian).

3. A.A. Torokin, Engineering-technical Protection of Information. Helios ARV, Moscow, 2005 (in Russian).

4. V.F. Venda, Ergonomics. Mir, Moscow, 1971 (in Russian).

5. V.F. Venda, Engineering Psychology and Synthesis of the Information Display Systems. Mashinostroyeniye, Moscow, 1975 (in Russian).

6. V.I. Kostyuk, V.E. Khodakov, Systems for Information Display and Engineering Psychology. Vyshcha shkola, Kiev, 1977 (in Russian).

7. F.M. Yablonski, Yu.V. Troitski, Means for Information Display. Vysshaya shkola, Moscow, 1985 (in Russian).

8. A.A. Samarsky, A.P. Mikhailov, Mathematical Modeling: Ideas. Methods. Examples. Nauka, Moscow, 1997 (in Russian).

9. B.Ya. Sovetov, S.A. Yakovlev, Modeling Systems. Vysshaya shkola, Moscow, 2001 (in Russian).

10. Introduction to Mathematical Modeling, Edited by P.V. Trusov. Logos, Moscow, 2005 (in Russian).

11. A.D. Myshkis, Elements of the Theory of Mathematical Models. Komkniga, Moscow, 2007 (in Russian).

12. V.M. Gasov, A.V. Men'kov, L.A. Solomonov, A.V. Shigin, System Designing Interaction of a Man with Technical Means, Book 7 // Organization of Human Interaction with Technical Means of $A C S$, Edited by V.N. Chetverikov, In 7 books. Vysshaya shkola, Moscow, 1990 (in Russian).

13. Applied Problems of Systemotechnics, Edited by A.V. Il'ichov. Mashinostroyeniye, Moscow, 1995 (in Russian). 